\title{
Desmoplastic Ameloblastoma of the Mandible
}

\author{
Soniya Adyanthaya, ${ }^{1}$ Pushparaja Shetty ${ }^{2}$ \\ 'Department of Oral Pathology and Microbiology, Yenepoya Dental College, Karnataka State, India, ${ }^{2}$ Department of Oral \\ Pathology and Microbiology, A.B. Shetty Memorial Institute of Dental Sciences, Karnataka State, India.
}

\section{ABSTRACT}

The Desmoplastic Ameloblastoma exhibits important differences in anatomical distribution, histologic appearance and radiographic findings compared with the classic type of ameloblastoma. We describe a case of desmoplastic variant of ameloblastoma localized in the mandibular anterior portion of the jaw, extending posteriorly in the left side. The tumor was expansile, slowly growing and painless. The radiographic finding showed diffuse, radiolucent lesion with cortical bone expansion. Surgical excision was performed and follow-up examination done after two years disclosed no recurrence. The purpose of this article is to assist in better understanding of this variant of odontogenic tumor.

Keywords: ameloblastoma; desmoplasia; mandible; odontogenic tumor.

\section{INTRODUCTION}

Ameloblastoma is a benign epithelial odontogenic tumor that usually exhibits aggressive behavior, causes severe expansion of the cortical bones and may have a high recurrence rate. ${ }^{1}$ This tumor occurs most frequently in the posterior mandible, and usually appears as a well-demarcated unilocular or multilocular radiolucent lesion. ${ }^{2}$ The histologic variants of ameloblastoma include follicular, plexiform, acanthomatous, granular cell, desmoplastic, basal cell, keratoameloblastoma, papilliferous and clear cell ameloblastoma. ${ }^{3} \mathrm{~A}$ desmoplastic variant with features of other histologic types is termed a "hybrid". ${ }^{4}$

The first detailed report on the desmoplastic variant of ameloblastoma in the English literature was given by Eversole et al in 1984 who called it an ameloblastoma with pronounced desmoplasia. ${ }^{5}$ Takigawa et al, ${ }^{6}$ and Uji et $\mathrm{al}^{7}{ }^{7}$ also described this lesion characterized by extensive stromal desmoplasia with small compressed nests and strands of odontogenic epithelium. It has been categorized as a distinct variant of ameloblastoma in the World Health Organization (WHO) classification of odontogenic tumors in 2003 due to its distinct clinicoradiographic presentation and pathology. ${ }^{8}$

The desmoplastic variant differs from other histologic types of ameloblastoma in that it is located in the anterior or premolar regions of the maxilla or mandible. Radiographically it resembles a fibro-osseous lesion because of its diffuse, mixed radiolucent-radiopaque appearence with a honeycomb or soap bubble pattern and indistinct borders. ${ }^{9,10}$ Histopathologically, desmoplastic ameloblastomas are nonencapsulated tumours with extensive collagenous stroma or desmoplasia containing small odontogenic epithelium islands and cords of various sizes. ${ }^{2,11}$ In view of the paucity of Desmoplastic Ameloblastoma (DA) case

Correspondence: Dr. Soniya Adyanthaya, Department of Oral Pathology and Microbiology, Yenepoya Dental College, Mangalore-575018, Karnataka State, India. Email: drsoniya1978@gmail.com, Phone: +919741231333 
series and the only limited understanding of its biologic behavior and prognosis, the proper treatment strategies for DA are not entirely defined so far. ${ }^{12}$ This report describes a case of desmoplastic ameloblastoma in the mandibular jaw of a male patient.

\section{CASE REPORT}

A 40 year-old man visited oral and maxillofacial surgery clinic for evaluation and treatment of an expansile lesion in the mandibular region extending form left mandibular first molar region to mandibular right canine. The lesion had been present for eight months. He revealed a history of a slowly enlarging mandibular mass, without pain or bleeding. The patient had undergone extraction of mandibular lateral incisor, canine, first and second premolar and first molar few years back due to periodontal problems. The patient's medical history did not reveal any significant finding.

The intraoral examination disclosed a large, hard, firm, non tender mass of the lower jaw causing buccal and lingual cortical expansion. The lesion was covered by an intact overlying mucosa. Grade I mobility was noted in mandibular left central incisor and mandibular right central incisor and lateral incisor. No lymphadenopathy or fistulae were present.

Panoramic radiography revealed a diffuse, multilocular radiolucent lesion from left mandibular first molar, crossing the midline and extending up to right mandibular canine region (Figure 1).

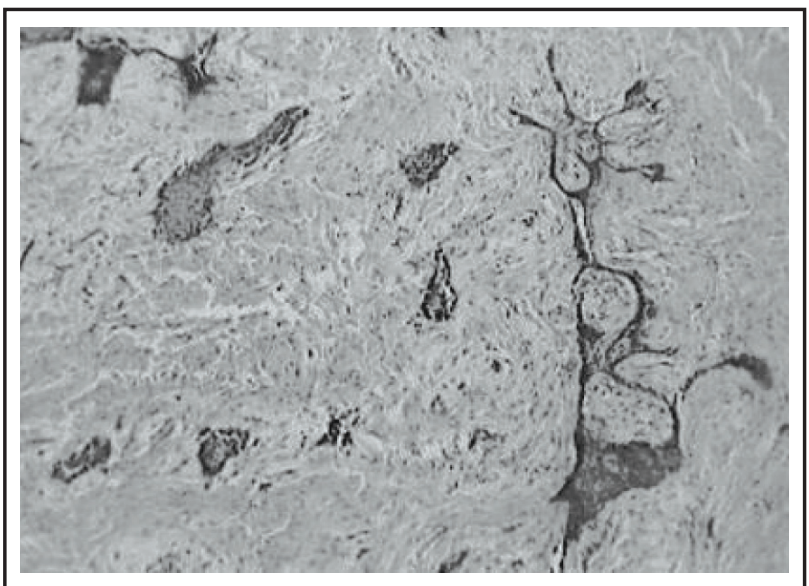

Figure 1. Panoramic radiograph shows expansile, radiolucent lesion with diffuse borders extending form left first molar region to right mandibular canine.

The clinical differential diagnosis included ameloblastoma and odontogenic keratocyst. The incisional biopsy was performed under local anaesthesia. The microscopic examination revealed odontogenic epithelial islands and cords surrounded by a dense, hypocellular, fibrous, connective tissue that appears to compress the odontogenic islands. The diagnosis of desmoplastic ameloblastoma was established.

The partial mandibulectomy with extraction of left mandibular central incisor, right mandibular central incisor, lateral incisor, and canine followed by reconstruction of the defect and primary mucosal closure was performed. Histopathological examination revealed scattered odontogenic epithelial islands arranged in cords and strands showing 'animal' pattern in a dense fibrous connective tissue stroma (Figure 2, 3). These islands were formed of flattened peripheral cells and central polygonal or spindle-shaped cells. The diagnosis of desmoplastic ameloblastoma was made.

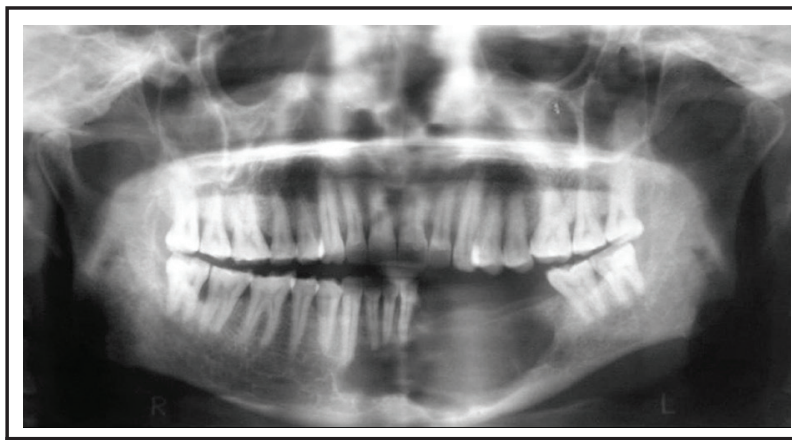

Figure 2. Odontogenic epithelial islands and cords in a dense fibrous connective tissue stroma with a stellate or an "animal-like" configuration. (Hematoxylin-eosin stain, $40 x$ ).

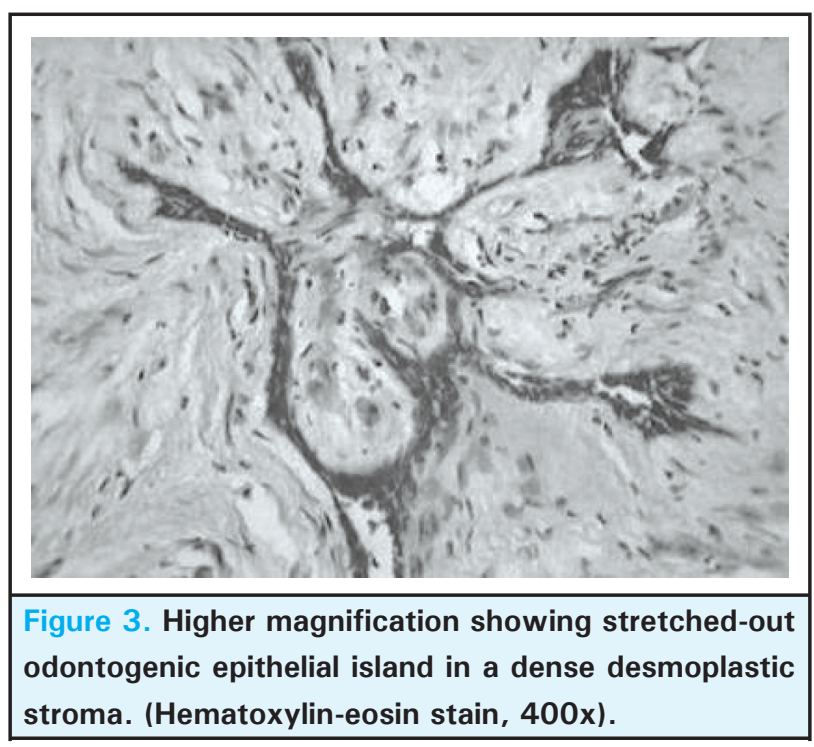

The patient's postoperative course was uneventful. A permanent prosthesis was provided at the oneyear postoperative appointment. A clinical follow-up examination two years later disclosed no recurrence or 
residual tumor.

\section{DISCUSSION}

The desmoplasic ameloblastoma was firstly described by Eversole et al in $1984 .{ }^{10}$ The clinical, radiographic and histopathologic fea tures of 145 cases reported in Japanese, Chinese, Malaysian, Western, and African populations, with very few cases of Indians has been reviewed. ${ }^{13} \mathrm{~A}$ retrospective study of 115 cases of desmoplastic ameloblastoma reported in literature from 1984 to 2008 concluded that it presents distinct clinical, radiographic and histologic features when compared to "conventional ameloblastomas". ${ }^{12}$

Approximately half of the desmoplastic lesions are located in the maxilla, and the vast majority of them occur in the anterior or premolar portion of the jaws. Frequently, the tumor is asymptomatic, the tumefaction is painless and expansion of the involved bone is the commonly observed clinical manifestations. ${ }^{14}$

In a review of 115 cases of desmoplastic ameloblastomas, the commonly observed radiographic features were mixed radiolucent/radiopaque (56\%), multilocular (49\%), and with ill-defined borders $(64 \%) .{ }^{12}$ In the present case, multolocular radiolucency was observed in the mandibular anterior region.

Histopathologically, desmoplastic ameloblastoma is characterized by small nests and cords of odontogenic tumoral epithelium, organized in an abundant stroma densely collagenized, which makes these tumoral islands seem like they are compressed. The peripheral layer of the cells is usually cuboidal and occasionally hyperchromatic. ${ }^{14}$ The morphologic characteristics typical of multicystic ameloblastomas such as the polarity inversion and typical palisade pattern are not observed frequently. The central portion of the tumoral islands resembling stellate reticulum is occasionally encountered. This area appears hypercellular and is composed of spindle- shaped or polygonal epithelial cells. ${ }^{15}$ The histopathological characteristics in this case are in accordance to the above diagnostic features.

It has been proposed that the extensive desmoplasia observed in this tumor has its origin from a new protein synthesis that comes from the extracellular matrix. ${ }^{16}$ In the desmoplastic variation, the presence of an osseous neo-formation, which probably contributes to the radiopacity, may be prominent in some areas. ${ }^{15}$ In the above case, such osseous formation was not evidenced in the epithelial proliferation.

The recurrence level of the desmoplasic variant is still not known due to the low number of reported cases in the literature. As the tumour is non-encapsulated, the cells infiltrate between the trabeculae of the cancellous bone leaving them intact for some time. Thus, the tumour actually extends beyond the radiographic margin $^{2}$ which could be the reason for the ill defined radiographic borders and the high recurrence rate after curettage.

Due to the lack of capsule and precise limits, a radical treatment is recommended for most of the desmoplastic ameloblastoma cases. ${ }^{14}$ However, because of the limited understanding on the biological and prognostic behavior, the therapeutical approach strategy is still not completely clear. In the present case, no recurrence was observed in two years of follow up period.

\section{CONCLUSIONS}

The treatment modalities, behavior and the eventual prognosis of desmoplastic ameloblastomas still cannot be predicted with certainty because of limited number of reported cases. Desmoplastic ameloblastoma will remain an enigma until researchers pursue more definitive tumor analysis techniques, long term followup and reporting in many more cases.

\section{REFERENCES}

1. Gardner DG, Pecak AM. The treatment of ameloblastoma based on pathologic and anatomic principles. Cancer. 1980;46:2514-9.

2. Gardner DG. Some current concepts on the pathology of ameloblastomas. Oral Surg Oral Med Oral Pathol Oral Radiol Endod. 1996;82:660-9.

3. Waldron CA, el-Mofty SK. A histopathologic study of $\mathbf{1 1 6}$ ameloblastomas with special reference to the desmoplastic variant. Oral Surg Oral Med Oral Pathol. 1987;63:441-51.

4. Ashman SG, Corio RL, Eisele DW, Murphy MT. Desmoplastic ameloblastoma. A case report and literature review. Oral Surg Oral Med Oral Pathol. 1993;75(4):479-82.

5. Eversole LR, Leider AS, Hansen LS. Ameloblastomas with pronounced desmoplasia. J Oral Maxillofac Surg. 1984;42:735-40.

6. Takigawa T, Matsumoto M, Sekine Y. A case report of ameloblastoma proliferated like epulis of maxilla [in Japanese]. Nihon Univ Dent. 1981;55:920-4. 
7. Uji Y, Kodama K, Sakamoto A, Taen A. An ameloblastoma with interesting findings [in Japanese]. Jpn J Oral Maxillofac Surg. 1983;29:1512-9.

8. Barnes L, Eveson JW, Reichart PA, Sidransky D. World Health Organization Classification of Tumors. Pathology and Genetics of Head and Neck Tumors. Lyon: IARC Press; 2005:284.

9. Kawai T, Kishino M, Hiranuma H, Sasai T, Ishida T. A unique case of desmoplastic ameloblastoma of the mandible: report of a case and brief review of the English language literature. Oral Surg Oral Med Oral Pathol Oral Radiol Endod. 1999;87(2):258-63.

10. Yazdi I DMD, Seyedmajidi M, Foroughi R. Desmoplastic Ameloblastoma (a Hybrid Variant): Report of a Case and Review of the Literature. Arch Iranian Med. 2009;12(3):304-8.

11. Pillai RS, Ongole R, Ahsan A, Radhakrishnan RA, Pai KM. Recurrent Desmoplastic Ameloblastoma of the Maxilla: A Case Report. J Can Dent Assoc. 2004;70(2):100-4.
12. Sun ZJ, Wu YR, Cheng N, Zwahlen RA, Zhao YF, Desmoplastic ameloblastoma - A review. Oral Oncology. 2009;45(9):752-9.

13. Sivapathasundharam B, A Einstein A, Syed RI. Desmoplastic ameloblastoma in Indians: Report of five cases and review of literature. Indian J Dent Res. 2007;18:218-21.

14. Alves PM, Pereira KMA, Vasconcelos MG, Souza LB, Queiroz LMG, Medeiros AM. Desmoplasic Ameloblastoma in Maxilla - Report of Case and Review of the Literature. Int. J. Morphol. 2008;26(2):263-8.

15. Shashikanth MC, Neetha MC, Ali IM, Shambulingappa P. Desmoplastic ameloblastoma in the maxilla: A case report and review of literature. Indian J of Dent Res. 2007;18(4):214-7.

16. Phillipsen HP; Reichart PA;T Takata. Desmoplastic ameloblastoma (including "hybrid" lesion of ameloblastoma). Biological profile based on 100 cases from the literature and own files. Oral Oncol. 2001;37(5):455-60. 